\title{
A MEASURE THEORETICAL SUBSEQUENCE CHARACTERIZATION OF STATISTICAL CONVERGENCE
}

\author{
HARRY I. MILLER
}

\begin{abstract}
The concept of statistical convergence of a sequence was first introduced by $\mathrm{H}$. Fast. Statistical convergence was generalized by R. C. Buck, and studied by other authors, using a regular nonnegative summability matrix $A$ in place of $C_{1}$.

The main result in this paper is a theorem that gives meaning to the statement: $S=\left\{s_{n}\right\}$ converges to $L$ statistically $(T)$ if and only if "most" of the subsequences of $S$ converge, in the ordinary sense, to $L$. Here $T$ is a regular, nonnegative and triangular matrix.

Corresponding results for lacunary statistical convergence, recently defined and studied by J. A. Fridy and C. Orhan, are also presented.
\end{abstract}

\section{INTRODUCTION}

The concept of the statistical convergence of a sequence of reals $S=\left\{s_{n}\right\}$ was first introduced by $\mathrm{H}$. Fast [9].

The sequence $S=\left\{s_{n}\right\}$ is said to converge statistically to $L$ and we write

$$
\begin{gathered}
\lim _{n \rightarrow \infty} s_{n}=L \text { (stat) if for every } \varepsilon>0, \\
\lim _{n \rightarrow \infty} n^{-1}\left|\left\{k \leq n:\left|s_{k}-L\right| \geq \varepsilon\right\}\right|=0,
\end{gathered}
$$

where $|A|$ denotes the cardinality of the set $A$.

Properties of statistically convergent sequences were studied in $[5,6,12$, and 16]. In [13] Fridy and Miller gave a characterization of statistical convergence for bounded sequences using a family of matrix summability methods.

Statistical convergence can be generalized by using a regular nonnegative summability matrix $A$ in place of $C_{1}$. This idea was first mentioned by R. C. Buck [3] in 1953 and has been further studied by Sember and Freedman ([10 and 11]) and Connor ([5 and 7]). Regular nonnegative summability matrices turn out to be too general for our purposes here, instead we use the concept of a mean.

A matrix $T=\left(a_{m n}\right)$ will be called a mean if $a_{m n}>0$ when $n \leq m, a_{m n}=0$ if $n>m, \sum_{n=1}^{\infty} a_{m n}=1$ for all $m$ and $\lim _{m \rightarrow \infty} a_{m n}=0$ for each $n$.

If $T=\left(a_{m n}\right)$ is a mean, following Buck, a sequence $S=\left\{s_{n}\right\}$ is said to be statistically $T$-summable to $L$ and we write

$$
s_{n} \rightarrow L(\text { stat } T) \text { if for every } \varepsilon>0
$$

Received by the editors August 18, 1993 and, in revised form, February 14, 1994; originally communicated to the Proceedings of the AMS by Andrew Bruckner.

1991 Mathematics Subject Classification. Primary 40D25; Secondary 40G99, 28 A12. 
we have

$$
\sum_{n=1}^{\infty}\left[a_{m n}:\left|s_{n}-L\right| \geq \varepsilon\right] \rightarrow 0 \quad \text { as } m \rightarrow \infty .
$$

The main result in this paper is a theorem that gives meaning to the statement $S=\left\{s_{n}\right\}$ converges to $L$ statistically $(T)$ if and only if "most" of the subsequences of $S$ converge, in the ordinary sense, to $L$.

In [14] and [15] Fridy and Orhan studied lacunary statistical convergence. We will present a measure theoretical subsequence characterization of lacunary statistical convergence.

Results. We recall that Fridy proved [12] that a sequence $S$ is statistically convergent if and only if there exists a subset $A$ of $\mathbb{N}$ (the natural numbers), having density zero, such that the subsequence of $S$ obtained by removing the terms of $S$ with indices in $A$ is convergent in the ordinary sense. Here, $A$ having density zero means

$$
\lim _{n \rightarrow \infty} n^{-1}|\{k \leq n: k \in A\}|=0
$$

Our first step toward obtaining a subsequence characterization of statistical $(T)$ convergence is the following generalization of the result of Fridy just mentioned. In the statement of our theorem we will need a definition of $T$-density zero.

If $T=\left(a_{m n}\right)$ is a mean, then a subset $A$ of $\mathbb{N}$ is said to have $T$-density zero if

$$
\lim _{m \rightarrow \infty} \sum_{n \in A} a_{m n}=0
$$

Theorem 1. $s_{n} \rightarrow L$ (stat $T$ ) if and only if there is a subset $A$ of $\mathbb{N}$ such that $s_{n_{k}} \rightarrow L$ (in the usual sense) as $k \rightarrow \infty$, where $\mathbb{N} \backslash A=\left\{n_{k}: k \in \mathbb{N}\right\}$ and $A$ has $T$-density zero.

Proof. Suppose $s_{n} \rightarrow L($ stat $T)$. For each $\varepsilon_{n}=\frac{1}{n}, n=2,3, \ldots$, there exists a positive integer $r_{n}$ (with the sequence $\left\{r_{n}\right\}_{n=2}^{\infty}$ strictly increasing) such that

$$
r \geq r_{n} \text { implies } \sum_{k=1}^{\infty}\left[a_{r k}:\left|s_{k}-L\right| \geq \frac{1}{n}\right]<\frac{1}{n^{2}} .
$$

Set,

$$
A=\bigcup_{n=2}^{\infty}\left\{k: r_{n} \leq k<r_{n+1} \text { and }\left|s_{k}-L\right| \geq \frac{1}{n}\right\} .
$$

The subsequence of $S$ obtained by removing the terms with indices in $A$ clearly converges, in the ordinary sense, to $L$.

We will now show that $A$ has $T$-density zero. Let $\varepsilon>0$. There exists an $n(\varepsilon) \in \mathbb{N}$ such that

$$
\sum_{n=n(\varepsilon)}^{\infty} \frac{1}{n^{2}}<\frac{\varepsilon}{2}
$$

The regularity of $T$ implies there exists an $R_{n(\varepsilon)}$, a term in the sequence $\left\{r_{n}\right\}$ with index larger than $n(\varepsilon)$, i.e., $R_{n(\varepsilon)}=r_{m(\varepsilon)}, m(\varepsilon)>n(\varepsilon)$, such that

$$
\sum_{i=1}^{r_{n(\varepsilon)}-1} a_{r i}<\frac{\varepsilon}{2} \text { for all } r \geq R_{n(\varepsilon)}\left(=r_{m(\varepsilon)}\right) \text {. }
$$


Now suppose $r \geq r_{m(\varepsilon)}$. We have

$$
\sum_{i \in A} a_{r i} \leq \sum_{i<r_{n(\varepsilon)}} a_{r i}+\sum_{\substack{i \in A \\ i \geq r_{n(e)}}} a_{r i}
$$

with $r \geq r_{m(\varepsilon)}>r_{n(\varepsilon)}, r_{j} \leq r<r_{j+1}$, so

$$
\begin{aligned}
\sum_{\substack{i \in A \\
i \geq r_{n(\varepsilon)}}} a_{r i}=\sum_{\substack{i \in A \\
r_{n}(\varepsilon) \leq i \leq r_{n(\varepsilon)+1}}} a_{r i}+\sum_{\substack{i \in A \\
r_{n(\varepsilon)+1} \leq i<r_{n(\varepsilon)+2}}} a_{r i}+\cdots+\sum_{\substack{i \in A \\
r_{j} \leq i<r_{j+1}}} a_{r i} \\
<\frac{1}{n^{2}(\varepsilon)}+\frac{1}{(n(\varepsilon)+1)^{2}}+\cdots+\frac{1}{j^{2}}<\sum_{n=n(\varepsilon)}^{\infty} \frac{1}{n^{2}}<\frac{\varepsilon}{2}
\end{aligned}
$$

by $(*)$ and the definition of $A$. So, by (**)

$$
\sum_{i \in A} a_{r i}<\frac{\varepsilon}{2}+\frac{\varepsilon}{2} \quad \text { if } r \geq r_{m(\varepsilon)}
$$

or $A$ has $T$-density zero.

Now we look at the converse. Suppose that $A$ has $T$-density zero, $\left\{n_{k}\right\}=$ $\mathbb{N} \backslash A$ and $s_{n_{k}} \rightarrow L$ (in the ordinary sense). We must show that $s_{n} \rightarrow L$ (stat $T$ ). Let $\varepsilon>0$. Then there exists a $k(\varepsilon)$ such that $k \geq k(\varepsilon)$ implies $\left|s_{n_{k}}-L\right|<\varepsilon$ and therefore

$$
\begin{aligned}
\sum_{k}[ & \left.a_{r k}:\left|s_{k}-L\right| \geq \varepsilon\right] \\
& \leq \sum_{k<n_{k(\varepsilon)}} a_{r k}+\sum_{k \geq n_{k(\varepsilon)}}\left[a_{r k}:\left|s_{k}-L\right| \geq \varepsilon\right] \\
& \leq \sum_{k<n_{k(\varepsilon)}} a_{r k}+\sum\left[a_{r k}: k \in A\right] .
\end{aligned}
$$

$\lim _{r \rightarrow \infty} \sum_{k<n_{k}(\varepsilon)} a_{r k}=0$ since $T$ is regular and $\lim _{r \rightarrow \infty} \sum\left[a_{r k}: k \in A\right]=0$ because $A$ has $T$-density 0 .

We now observe that there is a one-to-one onto correspondence between the interval $(0,1]$ and the collection of all subsequences of the sequence $S=$ $\left\{s_{n}\right\}$. Namely, if $x \in(0,1]$, then $x$ has a unique binary expansion $x=$ $\sum_{n=1}^{\infty} e_{n}(x) 2^{-n}, e_{n}(x) \in\{0,1\}$, with infinitely many ones. For each $x \in$ $(0,1]$, let $S(x)$ denote the subsequence of $S$ obtained by the following rule: $s_{n}$ is in the subsequence $S(x)$ if and only if $e_{n}(x)=1$. Clearly the mapping $x \mapsto S(x)$ is a one-to-one onto mapping between $(0,1]$ and the collection of all subsequences of $S$.

Suppose $T$ is a mean and $s_{n} \rightarrow L($ stat $T)$. It is natural to consider the set

$$
C_{L}:=\{x \in(0,1]: S(x) \text { converges to } L\} \text {. }
$$

This set may well have Lebesgue measure zero as the following example shows and hence "most" of the subsequences of $S=\left\{s_{n}\right\}$, in the sense of Lebesgue measure, need not converge to $L$.

Example 1. Let $T$ denote the $(C, 1)$ matrix and hence (stat $T$ ) convergence is statistical convergence. Let $A$ be any infinite subset of $\mathbb{N}$ having density 
zero. Define $S=\left\{s_{n}\right\}$ as follows. $s_{n}=1$ if $n \notin A$ and $s_{n}=0$ if $n \in A$. Two easy applications of Borel's normal number theorem [2, p. 9] shows that

$$
\begin{aligned}
m(\{x \in(0,1]: S(x) \text { has infinitely many zero terms and } \\
\text { infinitely many one terms }\})=1
\end{aligned}
$$

where $m$ is Lebesgue measure. Also, $\lim _{n \rightarrow \infty} s_{n}=1$ (stat).

This example shows that to get the theorem mentioned in the introduction it will be necessary to use a measure different from Lebesgue measure.

In the following, if $A=\left\{k_{n}\right\}$ is any subset of $\mathbb{N}, m_{A}$ will denote the unique probability measure defined on the Borel subsets of $(0,1]$ having the following property:

$$
m_{A}\left(\left\{x \in(0,1]: e_{j}(x)=1\right\}\right)= \begin{cases}\frac{1}{2} & \text { if } j \notin A, \\ \frac{1}{2^{n}} & \text { if } j=k_{n}\end{cases}
$$

and $\left\{e_{n}(x)\right\}_{n=1}^{\infty}$ is a sequence of independent random variables with respect to $m_{A}$. See [1].

\begin{tabular}{|c|c|c|c|c|c|}
\hline $2^{1}$ & half & closed & intervals & of & length \\
\hline $2^{2}$ & half & closed & intervals & of & length \\
\hline • & ․ & • & - & - & • \\
\hline • & • & - & - & - & - \\
\hline & . & . & • & $\cdot$ & · \\
\hline
\end{tabular}

To get a little better feel for $m_{A}$, consider the following inductive process. Suppose $m_{A}$ has been defined for the

Each of the last-mentioned $2^{j-1}$ intervals $I$ is divided into two abutting halfclosed intervals of length $\frac{1}{2^{j}}$, call them $I$ (left) and $I$ (right), the domain of $m_{A}$ is extended as follows:

$$
\begin{array}{rlrl}
m_{A}(I(\text { left })) & =\frac{1}{2} m_{A}(I) & \\
m_{A}(I(\text { right })) & =\frac{1}{2} m_{A}(I) & & \\
m_{A}(I(\text { left })) & =\left(1-\frac{1}{2^{n}}\right) m_{A}(I) & \\
m_{A}(I(\text { right })) & =\frac{1}{2^{n}} m_{A}(I) . & \left(\text { if } j=k_{n}\right) .
\end{array}
$$

$m_{A}$ is the unique probability measure on the Borel subset of $(0,1]$ whose values on half-closed dyadic subintervals are given above.

The purpose of using $\frac{1}{2^{n}}$ instead of $\frac{1}{2}$ when $j=k_{n}$ is to avoid "picking" elements of the "bad" set $A$.

We are now ready to prove the main result in this paper.

Theorem 2. Suppose $T=\left(a_{m n}\right)$ is a mean. The sequence $S=\left\{s_{n}\right\}$ converges (stat $T$ ) to $L$ (i.e., $s_{n} \rightarrow L($ stat $T)$ ) if and only if there exists a subset $A$ of $\mathbb{N}$ having $T$-density zero such that

$$
m_{A}\left(C_{L}\right)=m_{A}\left(\left\{x \in(0,1]: \lim _{n \rightarrow \infty}(S(x))_{n}=L\right\}\right)=1 .
$$

Proof. Suppose $s_{n} \rightarrow L$ (stat $\left.T\right)$. Then, by Theorem 1 , there exists a subset $A$ of $\mathbb{N}$, having $T$-density zero such that $\left\{x_{n_{k}}\right\}$ converges, in the ordinary sense, to $L$, where $\left\{n_{k}: k \in \mathbb{N}\right\}=\mathbb{N} \backslash A$. 
Notice that $S(x)$ converges to $L$ if $\left\{n \in A: e_{n}(x)=1\right\}$ is a finite set. However, by the first part of the Borel-Cantelli Lemma (see [2, p. 46])

$$
m_{A}\left(\left\{x \in(0,1]:\left\{n \in A: e_{n}(x)=1\right\} \text { is infinite }\right\}\right)=0
$$

since $\sum_{n \in A} m_{A}\left\{x \in(0,1]: e_{n}(x)=1\right\}=\sum_{n=1}^{\infty} \frac{1}{2^{n}}$ is convergent. Therefore $m_{A}\left(C_{L}\right)=1$.

Suppose now that $S=\left\{s_{n}\right\}$ is not statistically $(T)$ convergent and $A$ is any subset of $\mathbb{N}$ having $T$-density zero. Then, by Theorem $1\left\{s_{n_{k}}\right\}$, where $\left\{n_{k}\right\}=\mathbb{N} \backslash A$, does not converge. Then we have either

$$
\lim _{j \rightarrow \infty} s_{n_{k_{j}}}=+\infty \text { for some subsequence }\left\{n_{k_{j}}\right\} \text { of }\left\{n_{k}\right\}
$$

or

$$
\lim _{j \rightarrow \infty} s_{n_{k_{j}}}=-\infty \text { for some subsequence }\left\{n_{k_{j}}\right\} \text { of }\left\{n_{k}\right\}
$$

or there exist $\lambda<\mu$ and two infinite subsets $B$ and $C$ of $\mathbb{N}$ such that $A \cap B=$ $A \cap C=B \cap C=\varnothing$ and $s_{n}<\mu$ if $n \in B$ and $s_{n}>\mu$ if $n \in C$.

Now, since $m_{A}\left(\left\{x \in(0,1]: e_{n}(x)=1\right\}\right)=\frac{1}{2}$ if $n \notin A$, we have

$$
\begin{array}{ll}
\text { In Case 1, } & \sum_{j=1}^{\infty} m_{A}\left(\left\{x \in(0,1]: e_{n_{k_{j}}}(x)=1\right\}\right)=\sum \frac{1}{2}=\infty . \\
\text { In Case 2, } & \sum_{j=1}^{\infty} m_{A}\left(\left\{x:(0,1]: e_{n_{k_{j}}}(x)=1\right\}\right)=\sum \frac{1}{2}=\infty . \\
\text { In Case 3, } & \sum_{n \in B} \frac{1}{2}=\infty=\sum_{n \in C} \frac{1}{2}=\infty .
\end{array}
$$

So, by the second part of the Borel-Cantelli Lemma [2, p. 48] we have:

In Case $1, m_{A}\left(\left\{x \in(0,1]: e_{n_{k_{j}}}(x)=1\right.\right.$ for infinitely many $\left.\left.j\right\}\right)=1$.

In Case 2, $m_{A}\left(\left\{x \in(0,1]: e_{n_{k_{j}}}(x)=1\right.\right.$ for infinitely many $\left.\left.j\right\}\right)=1$.

In Case 3, $m_{A}\left(\left\{x \in(0,1]: e_{n}(x)=1\right.\right.$ for infinitely many $n \in B$ and also for infinitely many $n \in C\})=1$.

Therefore, in each of the above three cases we have

$$
m_{A}(\{x \in(0,1]: S(x) \text { is convergent }\})=0 \text {. }
$$

Example 1 shows that $s_{n} \rightarrow L($ stat $T)$, where $S=\left\{s_{n}\right\}$ is a sequence and $T$ is a mean, does not imply that

It is natural to ask if

$$
m(\{x \in(0,1]: S(x) \text { is convergent }\})=1 .
$$

$(*) \quad s_{n} \rightarrow L($ stat $T)$ implies $m\left(\left\{x \in(0,1]:(S(x))_{n} \rightarrow L(\right.\right.$ stat $\left.\left.T)\right\}\right)=1$.

It is easy to construct examples to show that $(*)$ does not hold in general.

A matrix summability method is said to have the Borel property if it sums “almost all" sequences of 0's and 1's to the value $\frac{1}{2}$; see [8, p. 207]. It is more involved to find a mean $T$ that has the Borel property and a sequence $S=\left\{s_{n}\right\}$ such that $s_{n} \rightarrow L($ stat $T)$ but

$$
m\left(\left\{x \in(0,1]:(S(x))_{n} \rightarrow L(\text { stat } T)\right\}\right)=0 .
$$


Example 2. Let $S=\left\{s_{n}\right\}=\{1,0,1,0,1, \ldots\}$. Let $T$ be the mean defined in the following way. $a_{11}=1$ and for each row $m, m \geq 2$, spread the total weight $1-\frac{1}{m}$ equally in the odd columns and spread the total weight $\frac{1}{m}$ equally in the even columns. Of course, $a_{m n}=0$ if $n>m$. Then $T$ is a mean satisfying the Borel property (see $[6$, p. 211]) and

$$
s_{n} \rightarrow 1(\operatorname{stat} T) \text {. }
$$

To show that $m(\{x \in(0,1]: S(x)$ is (stat $T)$ convergent $\})=0$, we consider the sequences $\left\{X_{n}\right\}_{n=1}^{\infty}$ of random variables on the probability space $((0,1], \mathscr{B}, m)$, where $\mathscr{B}$ are the Borel subsets of $(0,1]$, that are defined in the following manner. For each $x$ in $(0,1]$ :

$$
\begin{aligned}
& X_{1}(x)= \begin{cases}1 & \text { if }(S(x))_{1} \neq(S(x))_{3} \\
0 & \text { if }(S(x))_{1}=(S(x))_{3}\end{cases} \\
& X_{2}(x)= \begin{cases}1 & \text { if }(S(x))_{5} \neq(S(x))_{7}, \\
0 & \text { if }(S(x))_{5}=(S(x))_{7},\end{cases}
\end{aligned}
$$

Set

$$
Y_{n}=\frac{X_{1}+X_{2}+\cdots+X_{n}}{n}
$$

A little reflection shows that there exists an $a>0$ such that:

$$
m\left(\left[X_{1}=0\right]\right)>a, \quad m\left(\left[X_{1}=1\right]\right)>a
$$

and for each $n \geq 2$ and $i_{1}, i_{2}, \ldots, i_{n+1} \in\{0,1\}$

$$
m\left(\left[X_{n}=i_{n+1} \mid X_{1}=i_{1}, \ldots, X_{n}=i_{n}\right]\right)>a .
$$

This implies that (see [3])

$$
m\left(\left\{x \in(0,1]: \liminf _{n \rightarrow \infty} Y_{n}(x)>0\right\}\right)=1 .
$$

Moreover, if $\liminf _{n \rightarrow \infty} Y_{n}(x)>0, S(x)$ is not convergent (stat $T$ ) and hence $m(\{x \in(0,1]: S(x)$ is (stat $T)$ convergent $\})=0$.

Despite the above example we do have a characterization of statistical convergence.

Theorem 3. The sequence $S=\left\{s_{n}\right\}$ converges statistically to $L$ (i.e., $\lim _{n \rightarrow \infty} s_{n}$ $=L($ stat $))$ if and only if

$$
m\left(\left\{x \in(0,1]: \lim _{n \rightarrow \infty}(S(x))_{n}=L \text { (stat) }\right\}\right)=1 .
$$

Proof. Suppose $\lim _{n \rightarrow \infty} s_{n}=L$ (stat) and $x \in(0,1]$ is a normal number, i.e., $\frac{1}{n} \sum_{k=1}^{n} e_{k}(x) \rightarrow \frac{1}{2}$ as $n \rightarrow \infty$. Then $S(x)=\left\{s_{n_{1}}, s_{n_{2}}, \ldots\right\}$ where $\lim _{k \rightarrow \infty} \frac{n_{k}}{k}=$ 2 . Let $\varepsilon>0$. Then

$$
\begin{aligned}
\frac{1}{k} \mid\{i & \left.\leq k:\left|s_{n_{i}}-L\right| \geq \varepsilon\right\}\left|\leq \frac{1}{k}\right|\left\{i \leq n_{k}:\left|s_{i}-L\right| \geq \varepsilon\right\} \mid \\
& =\frac{n_{k}}{k} \frac{\left|\left\{i \leq n_{k}:\left|s_{i}-L\right| \geq \varepsilon\right\}\right|}{n_{k}} \rightarrow 2 \cdot 0=0
\end{aligned}
$$


as $k \rightarrow \infty$. Therefore $\lim _{n \rightarrow \infty}(S(x))_{n}=L$ (stat) if $x$ is a normal number. Since $M=\{x:(0,1]: x$ is a normal $\}$ has Lebesgue measure one the proof of the forward implication is complete.

Conversely, assume

$$
\left.m\left(\left\{x \in(0,1]: \lim _{n \rightarrow \infty}(S(x))_{n}=L \text { (stat }\right)\right\}\right)=1 .
$$

Then there exist two disjoint subsets $\left\{n_{k}: k \in \mathbb{N}\right\}$ and $\left\{n_{k}^{\prime}: k \in \mathbb{N}\right\}$ of $\mathbb{N}$ such that:

(1) $\left\{n_{k}: k \in \mathbb{N}\right\} \cup\left\{n_{k}^{\prime}: k \in \mathbb{N}\right\}=\mathbb{N}$,

(2) $\lim _{k \rightarrow \infty} \frac{n_{k}}{k}=2=\lim _{k \rightarrow \infty} \frac{n_{k}^{\prime}}{k}$, and

(3) $s_{n_{k}} \rightarrow L$ (stat) $s_{n_{k}^{\prime}} \rightarrow L$ (stat).

These three properties imply that

$$
s_{n} \rightarrow L \text { (stat), completing the proof. }
$$

Remark 1. Suppose $0<c_{1}<1<c_{2}$ and $T=\left(a_{m n}\right)$ is a mean satisfying

(**) $\quad \frac{c_{1}}{m} \leq a_{m n} \leq \frac{c_{2}}{m}$ for each $m \in \mathbb{N}$ and $n=1,2,3, \ldots, m$.

Then $s_{n} \rightarrow L$ (stat) if and only if $s_{n} \rightarrow L$ (stat $T$ ). Therefore Theorem 3 can be extended to (stat $T$ ) convergence if $T$ satisfies $(* *)$.

Fridy and Orhan in [14] and [15] studied lacunary statistical convergence. By a lacunary sequence we mean an increasing sequence of positive integers $\theta=\left\{k_{r}\right\}$ such that $h_{r}: k_{r}-k_{r-1} \rightarrow \infty$ as $r \rightarrow \infty$. In the following we denote by $I_{r}:=\left(k_{r-1}, k_{r}\right]$. Let $\theta$ be a lacunary sequence; they defined the sequence of numbers $S=\left\{s_{n}\right\}$ to be $S_{\theta}$-convergent to $L$ provided for every $\varepsilon>0$,

$$
\lim _{r \rightarrow \infty} h_{r}^{-1}\left|\left\{k \in I_{r}:\left|s_{k}-L\right| \geq \varepsilon\right\}\right|=0
$$

and we write $s_{n} \rightarrow L\left(S_{\theta}\right)$.

The following result is an analogue of a theorem of Fridy for statistical convergence that can be found in [8] and related to Theorem 1 in this paper.

Theorem 4. The sequence $S=\left\{s_{n}\right\}$ satisfies $s_{n} \rightarrow L\left(S_{\theta}\right)$ for a lacunary sequence $\theta=\left\{k_{r}\right\}$ if and only if there exists a subset $A$ of the natural numbers such that the subsequence of $S$ obtained by removing the terms of $S$ with indices in $A$ converges to $L$ in the ordinary sense and $\lim _{r \rightarrow \infty}\left|A \cap I_{r}\right| \cdot h_{r}^{-1}=0$.

Proof. Suppose $A$ is a subset of $\mathbb{N}$ such that $\lim _{r \rightarrow \infty}\left|A \cap I_{r}\right| \cdot h_{r}^{-1}=0$ and the subsequence of $S$ obtained by removing the terms with indices in $A$ converges in the ordinary sense to $L$. Then given $\varepsilon>0$,

$$
h_{r}^{-1}\left|\left\{k \in I_{r}:\left|s_{k}-L\right| \geq \varepsilon\right\}\right| \leq h_{r}^{-1}\left|A \cap I_{r}\right|
$$

for sufficiently large $r$ and hence $s_{n} \rightarrow L\left(S_{\theta}\right)$.

Conversely, suppose $s_{n} \rightarrow L\left(S_{\theta}\right)$. Then there exists a strictly increasing sequence of positive integers $\left\{r_{n}\right\}$ such that

$$
h_{r}^{-1}\left|\left\{k \in I_{r}:\left|s_{k}-L\right| \geq \frac{1}{n}\right\}\right|<\frac{1}{n}
$$

for all $r \geq r_{n}$. 
Let

$$
A=\bigcup_{i=1}^{\infty}\left\{k \in \bigcup_{j=r_{i}}^{r_{i+1}-1} I_{j}:\left|s_{k}-L\right| \geq \frac{1}{i}\right\} .
$$

Then $\lim _{r \rightarrow \infty}\left|I_{r} \cap A\right| \cdot h_{r}^{-1}=0$ and the subsequence of $S=\left\{s_{n}\right\}$ obtained by removing the terms of $S$ with indices in $A$ converges in the ordinary sense to $L$.

The last theorem can be used to obtain a $S_{\theta}$-convergence analogue of the proof of Theorem 2. Namely, we have the following:

Theorem 5. The sequence $S=\left\{s_{n}\right\}$ satisfies $s_{n} \rightarrow L\left(S_{\theta}\right)$ for a lacunary sequence $\theta=\left\{k_{r}\right\}$ if and only if there exists a subset $A$ of $\mathbb{N}$ such that

$$
\lim _{r \rightarrow \infty}\left|I_{r} \cap A\right| \cdot h_{r}^{-1}=0
$$

and

$$
m_{A}\left(C_{L}\right)=m_{A}\left(\left\{x \in(0,1]: \lim _{n \rightarrow \infty}(S(x))_{n}=L\right\}\right)=1 .
$$

Proof. If $s_{n} \rightarrow L\left(S_{\theta}\right)$, then $m_{A}\left(C_{L}\right)=1$. This follows as in the first half of the proof of Theorem 2, using Theorem 4 .

Suppose now that $S_{\theta}=\left\{s_{n}\right\}$ is not $S_{\theta}$-convergent and $A$ is a subset of $\mathbb{N}$ satisfying $\lim _{r \rightarrow \infty}\left|I_{r} \cap A\right| \cdot h_{r}^{-1}=0$. Then, by Theorem 4 , the subsequence $\left\{s_{n_{k}}\right\}$ of $S$, where $\left\{n_{k}\right\}=\mathbb{N} \backslash A$, does not converge. The remainder follows exactly as in the corresponding part of the proof of Theorem 2 .

We conclude by giving an example to show that the $S_{\theta}$-convergence analogue of Theorem 3 is false.

Example 3. Suppose $\theta$ is a lacunary sequence such that $h_{2}=10, h_{4}=12, h_{6}=$ $14, \ldots$. Define $S=\left\{s_{n}\right\}$ as follows: $s_{n}=0$ if $n \in I_{r}$ and $r$ is odd. If $r$ is even, the terms of $\left\{s_{n}\right\}$ with indices in $I_{r}$ are $1,0,1,0, \ldots, 1,0$. The sequence $h_{1}, h_{3}, h_{5}, \ldots$ can be taken large enough, and increasing fast enough to guarantee:

$$
\begin{gathered}
m\left(x \in(0,1]: h_{1}^{-1}\left|\left\{k \in I_{1}:(S(x))_{k}=0\right\}\right|>.99\right)>.99 \\
m\left(x \in(0,1]: h_{2}^{-1}\left|\left\{k \in I_{2}:(S(x))_{k}=0\right\}\right|>.999\right)>.999
\end{gathered}
$$

By the first part of the Borel-Cantelli Lemma

$$
m\left(\left\{x \in(0,1]:(S(x))_{n} \rightarrow 0\left(S_{\theta}\right)\right\}\right)=1,
$$

but $s_{n} \nrightarrow 0\left(S_{\theta}\right)$.

\section{ACKNOWLEDGMENT}

I would like to thank Professor M. S. Ramanujan, whose.very kind suggestions led to a substantial improvement in the content and style of this paper. I would also like to thank Professor S. Lu for useful conversations. 


\section{REFERENCES}

1. P. Billingsley, Ergodic theory and information, Wiley, New York, 1965.

2. _ Probability and measure, Wiley, New York, 1979.

3. R. C. Buck, Generalized asymptotic density, Amer. J. Math. 75 (1953), 335-346.

4. Y. S. Chow and H. Teicher, Probability theory, independence, interchangeability, martingales, 2nd ed., Springer-Verlag, New York, 1978.

5. J. Connor, The statistical and strong p-Cesàro convergence of sequences, Analysis 8 (1988), 47-63.

6. __ On strong matrix summability with respect to a modulus and statistical convergence, Canad. Math. Bull. 32 (1989), 194-198.

7. __ Two valued measures and summability, Analysis 10 (1990), 373-385.

8. R. G. Cooke, Infinite matrices and sequence spaces, Dover, New York, 1955.

9. H. Fast, Sur la convergence statistique, Colloq Math. 2 (1951), 241-244.

10. A. R. Freedman and J. J. Sember, On summing sequences of 0's and 1's, Rocky Mountain J. Math. 11 (1981), 419-425.

11. _ Densities and summability, Pacific J. Math. 95 (1981), 293-305.

12. J. A. Fridy, On statistical convergence, Analysis 5 (1985), 301-313.

13. J. A. Fridy and H. I. Miller, A matrix characterization of statistical convergence, Analysis 11 (1991), 59-66.

14. J. A. Fridy and C. Orhan, Lacunary statistical convergence (to appear).

15. __ Lacunary statistical summability (to appear).

16. I. J. Schoenberg, The integrability of certain functions and related summability methods, Amer. Math. Monthly 66 (1959), 361-375.

Department of Mathematics, University of Michigan, ANn Arbor, Michigan 48109

Current address: Department of Mathematics, The University of Tennessee at Chattanooga, Chattanooga, Tennessee 37403-2598 